\title{
The critical flaw in the implementation of GPS tracking system in express bus industry
}

\begin{abstract}
Land Public Transport Commission (SPAD) in Malaysia has made it mandatory that GPS tracking systems installed in every express buses since 2008 so that the speed can be monitored and recorded based on real-time. Nonetheless, the effectiveness of the system is under question when Malaysia was startled by a series of bus accidents each killed close to 10 lives while several others were seriously injured. This study is undertaken to identify the critical flaw in the implementation of the GPS monitoring system and propose a way to improve the existing monitoring system. Result from the interview session on top management of three express bus companies has found that the real-time record-keeping, which is a corrective measure, have been carried out instead of real-time monitoring, a preventive measure. Therefore, the existing model of speed management needs to be remodeled to alert the bus driver and the passengers who really concern about their own safety. A device and its main features which exploit the real-time monitoring ability of GPS are proposed for effective and systematic implementation of this new model.
\end{abstract}

Keyword: Global positioning system; Road accident; Safety introduction; Speed management 